Rev. Biol. Neotrop. 10 (2): 25 - 32. 2013

\title{
IVERSIDADE DE ARTRÓPODES NOS ABRIGOS FOLIARES PRODUZIDOS POR GONIOTERMA SP. (LePIDOPTERA) EM RAMOS DE ROUPALA MON- tana Aubl. (Proteaceae) no cerrado do Brasil central
}

\author{
RicARDo V. KiLCA
}

Laboratório de Dendrologia e Fitossociologia, Universidade Federal de Santa Maria, Cx Postal 5096, CEP 97105-900. Santa Maria, Rio Grande do Sul. rvkilka@gmail.com

\section{Everton Tizo-Pedroso}

Universidade Estadual de Goiás, campus Morrinhos, Rua 14, 625, Jardim América, CEP 75650-000, Morrinhos, Goiás.

\section{Roselaine R. Zanini}

Departamento de Estatística, Universidade Federal de Santa Maria, Cidade Universitária, Prédio 13, sala 1205A, Santa Maria, Rio Grande do Sul.

Resumo: Gonioterma sp. é uma espécie monófaga de lepidóptero que constrói seus abrigos foliares (AFs) em Roupala montana, uma espécie arbórea comum na vegetação dos cerrados do Brasil central. No Parque Estadual de Caldas Novas (PESCAN), estado de Goiás, foi realizado um estudo que procurou investigar: 1) preferência do lepidóptero em construir seu AF de acordo com o tamanho da planta; 2) a composição, riqueza e as guildas dos artrópodes associados aos AFs; e, 3) possível associação entre o tamanho das plantas com a abundância dos artrópodes presentes nesses AFs. Os principais resultados revelaram que as mariposas fêmeas de Gonioterma sp. podem depositar seus ovos em $R$. montana com diferentes classes de tamanhos, porém com preferência por plantas de maior porte. Os AFs apresentaram uma elevada riqueza e abundância de artrópodes de diferentes guildas, onde os mais representativos foram os predadores. Foi verificado associação positiva entre o tamanho de $R$. montana e a ocorrência de determinadas guildas de artrópodes. O papel exercido por Gonioterma sp. como engenheiro de ecossistema ficou destacado pela importância dos abrigos foliares produzidos por este animal como refúgios para a fauna de artrópodes locais.

Palavras-chave: Abrigo foliar, Cerrado, Interação planta-herbívoro, Mariposas, Plantas hospedeiras.

Abstract: Gonioterma sp. is a species of monophagous moths that build leaf shelters (LS) in Roupala montana, a common plant in the cerrado vegetation of central Brazil. In State Park of Caldas Novas (PESCAN), state of Goiás, a study was conducted in order to investigate: 1) the Lepidoptera preference to build its LS according to the plant size, plant density and different vegetation types in the landscape; 2 ) the composition, richness and guilds of arthropods associated with LF and, 3) the possible association between plants size with abundance of arthropods into LS. The results showed the female moths of Gonioterma sp. deposit their eggs preferentially on larger plants. The shelters provide a favorable micro-habitat for the development of the arthropods once showed high abundance and richness of different guilds, where the predators were the most representative group. There was positive association between size of $R$. montana and the occurrence of some arthropod guilds. The role of Gonioterma sp. as ecosystem engineer was highlighted by the importance of its shelters as refuges for the local arthropods.

Key words: Cerrado, herbivore-plants interactions, host plant, Leaf shelter, Moths. 


\section{INTRODUÇÃO}

Larvas de mariposas e borboletas (Lepidoptera), em geral, são os principais consumidores da biomassa foliar em ecossistemas terrestres. Aproximadamente 18 famílias de lepidópteros constroem abrigos foliares (AFs) em plantas (Jones et al., 2002). Larvas de lepidópteros constroem tais estruturas em suas plantas hospedeiras utilizando folhas mortas e/ou folhas jovens, juntamente com seda, que servem para enrolar, dobrar e unir estas partes (Damman, 1993; Fornier et al., 2003). Esses abrigos desempenham inúmeras funções ao herbívoro na fase inicial do seu desenvolvimento, como a manutenção de um microclima mais favorável, o aumento na qualidade nutricional e como proteção contra inimigos naturais (Damman, 1993; Jones et al., 2002). Os abrigos foliares também podem favorecer indiretamente outras espécies de artrópodes que utilizam as mesmas plantas hospedeiras, seja como refúgio ou local de forrageio (Damman, 1993; Fukui, 2001).

A fauna de Lepidoptera do Cerrado brasileiro é bastante rica, sendo estimada em mais de 10.000 espécies (Diniz \& Morais, 1997). Alguns dos estudos de interação entre lepidópteros e suas plantas hospedeiras nesse bioma têm procurado descrever a abundância e riqueza de lepidópteros nas plantas hospedeiras (Diniz \& Morais, 1995; 1997), a associação destes insetos com estruturas particulares das plantas (p.ex. foIhas, flores e frutos) (Diniz \& Morais, 2002), sua biologia comportamental (Morais et al., 2005) e a especificidade do herbívoro com determinadas espécies de plantas (Diniz et al., 1999; Costa \& Varanda, 2002; Morais et al., 2005). No entanto, ainda faltam informações sobre, por exemplo, como a forma e a estrutura das plantas hospedeiras interferem no comportamento de construção dos AFs e como esses mediam as interações secundárias entre os artrópodes.

Roupala montana Aubl. (Proteaceae) é uma espécie arbórea (até $12 \mathrm{~m}$ altura), semidecídua, que possui uma ampla distribuição nas diversas fitofisionomias dos cerrados do Brasil central, sendo mais abundante em cerrado sentido restrito, sob latossolos vermelho-escuro (Felfili, 1997). Dezenas de espécies de lepidópteros consomem folhas de $R$. montana, sendo quatro dessas consideradas monófagas para essa espécie-vegetal (Bendicho-López et al., 2006). Este fato indica a relevância desta planta na manutenção da diversidade deste grupo de artrópodes. Gonioterma (Lepidoptera: Elachistidae) corresponde a um gênero com 33 espécies de pequenas lagartas restrita à América tropical (Heppner, 1984). Em R. montana, Morais observou uma espécie de Gonioterma que constroi AFs nas folhas dessa planta hospedeira (Morais, H.C. dados não publicados).
O presente estudo buscou investigar alguns aspectos da interação entre Gonioterma sp. e $R$. montana em área de cerrado do Parque Estadual da Serra de Caldas Novas, dando ênfase nas seguintes questões: 1) existe uma preferência do lepidóptero em construir o seu AF de acordo com o tamanho da planta?; 2) qual a composição, riqueza e as guildas dos artrópodes associados aos AFs?; e 3) se existe uma associação entre o tamanho das plantas com as guildas dos artrópodes presentes nos AFs?

\section{Material e Métodos}

O estudo foi desenvolvido no Parque Estadual da Serra de Caldas Novas (PESCAN) $\left(17^{\circ} 43^{\prime} \mathrm{S}\right.$ e $\left.48^{\circ} 40^{\prime} \mathrm{O}\right)$ entre os dias 30 de setembro e 02 de outubro de 2005, no início do período das chuvas. O PESCAN está localizado no município de Caldas Novas, sudeste do estado de Goiás, Centro-oeste do Brasil. Segundo a classificação de Köppen (1948), a região localiza-se em clima Aw, tropical de savana, megatérmico. A temperatura anual média fica em torno de $23^{\circ} \mathrm{C}$, com pouca variabilidade ao longo do ano. O regime pluvial e a umidade do ar são definidos em duas estações climáticas distintas, a chuvosa, que ocorre de outubro a abril, e a seca, de maio a setembro (EMBRAPA, 1982).

Foi estabelecido um transecto de $1.500 \mathrm{~m}$ que percorreu uma estrada em declive desde o platô até as escarpas sudoeste do PESCAN e abrangeu quatro classes de fitofisionomias e solos. Nesse transecto foram incluídos na amostragem todos os indivíduos de $R$. montana que se encontravam a cinco metros de distância das duas bordas da estrada, em direção ao interior da vegetação. $O$ transecto foi subdividido em 15 sub-unidades amostrais contiguas de $100 \mathrm{x}$ $10 \mathrm{~m}(1.000 \mathrm{~m} 2)$. Dentro dessas sub-unidades, os indivíduos de $R$. montana foram identificados visualmente e selecionados apenas aqueles que se encontravam dentro de três critérios de altura: (i) plantas grandes: árvores maiores que $2 \mathrm{~m}$ (até $7 \mathrm{~m}$ - plantas mais altas encontradas na área de estudo); (ii) plantas de porte médio: variando entre 1 e $2 \mathrm{~m}$ e, (iii) plantas pequenas: maiores que $0,4 \mathrm{~m}$ e menores que $1 \mathrm{~m}$. Registrou-se o número de plantas em cada categoria de altura. Foram quantificados os abrigos foliares de Gonioterma sp. em todos os indivíduos de $R$. montana que apresentaram abrigos em suas copas.

Para estimar a riqueza de artrópodes nos abrigos foliares foi previamente estabelecido o número de 30 abrigos coletados aleatoriamente dentro de cada sub-unidade, sendo amostrado 
apenas um abrigo para cada planta de $R$. montana. Dessa maneira, foi possível preservar a independência entre os abrigos foliares. Para sistematização das coletas dos AFs, a copa das árvores foi dividida em um quadrante visual, sendo o primeiro ponto de coleta aquele no sentido norte-leste. Na ausência de abrigos, os outros quadrantes foram examinados em sentido horário. Com a ocorrência de mais de um abrigo no mesmo quadrante, optou-se por coletar aquele de maior tamanho, pois poderia suportar maior número de artrópodes residentes.

Após coletados, os abrigos foram acondicionados em sacos plásticos identificados. Durante a triagem em laboratório, os abrigos foram desmontados e todas as folhas que compunham a estrutura do abrigo foram quantificadas a partir do número de pecíolos existentes. Posteriormente, os artrópodes encontrados nos AFs foram coletados e acondicionados em frascos com álcool $70^{\circ}$, para posterior identificação. Os artrópodes coletados nos AFs foram identificados ao nível de família, de morfoespécie e na respectiva guilda funcional.

O teste Anova seguido do teste de Tukey (post-hoc) foi empregado para constatar diferenças na densidade de plantas nas diferentes classes de tamanho nas 15 sub-unidades. O número médio de folhas utilizadas pela larva de Gonioterma sp. para a confecção dos AFs foi comparado entre as três diferentes classes de tamanhos das plantas por meio do teste de Kruskall-Wallis.

A composição, abundância e riqueza de artrópodes nos abrigos foliares foram contabilizadas diretamente no processo de triagem e apresentados seus valores absolutos. Foi avaliada a associação entre o tamanho das plantas com a abundância dos indivíduos em cada guilda de artrópode por meio da análise de correspondência.

\section{Resultados e discussão}

RELAÇÃO ENTRE ABUNDÂNCIA DOS ABRIGOS FOLIARES COM A ALTURA DE R. MONTANA

Das plantas de $R$. montana observadas, 73 eram de tamanho pequeno $(0,4 \mathrm{~m}<\mathrm{h}<1 \mathrm{~m})$, 85 de porte médio ( $1 \mathrm{~m} \leq \mathrm{h}<2 \mathrm{~m}$ ) e 17 eram grandes (h>2 m) (Tabela 1). A maioria dos indivíduos de R. montana não apresentou abrigos foliares (122 ind. ou $69,3 \%$ ind.), sendo registrados abrigos em apenas $30,7 \%$ das plantas (53 ind.). As plantas de menor tamanho apresentaram menor número de AFs de Gonioterma sp. em suas copas $(n=16)$ em relação às plantas de porte médio $(n=58)$ e grandes $(n=48)(K W=9,98$; $\mathrm{p}=0,006$ ) (Tabela 1 ). Predominou as plantas com um ou dois AFs em relação as plantas com grande densidade de AFs (Figura 1).

Tabela 1 - Características dos abrigos foliares de Gonioterma sp., herbívoro construtor, encontrados em $R$. montana de vegetação de cerrado no Parque Estadual de Caldas Novas, GO.

\begin{tabular}{|c|c|}
\hline Características & Valores \\
\hline Altura de $R$. montana & Média \\
\hline Plantas com tamanho grande $(n=17)$ & $1,3 \pm 1,3 b$ \\
\hline Plantas com tamanho médio $(n=85)$ & $5,6 \pm 3,6 a$ \\
\hline \multirow[t]{2}{*}{ Plantas com tamanho pequeno $(n=73)$} & $4,8 \pm 2,5 a$ \\
\hline & $F=7,26$ \\
\hline Estrutura dos AFs & Total de plantas \\
\hline Plantas com AFs & 53 \\
\hline \multirow[t]{2}{*}{ Plantas sem AFs } & 122 \\
\hline & Total de AFs \\
\hline Árvores grandes com AF $(n=12)$ e sem AF $(n=5)$ & $48 a$ \\
\hline Árvores médias com AF $(n=29)$ e sem AF $(n=56)$ & $58 a$ \\
\hline \multirow[t]{3}{*}{ Árvores pequenas com AF $(n=12)$ e sem AF $(n=61)$} & 16b \\
\hline & $K W=9,9$ \\
\hline & Média \\
\hline Média de folhas por AFs* & $10,5 \pm 5,2$ \\
\hline Média de folhas em plantas grandes $(n=8)$ & $9,3 \pm 5,8$ \\
\hline Média de folhas em plantas médias $(n=18)$ & $11,6 \pm 8,1$ \\
\hline \multirow[t]{2}{*}{ Média de folhas em plantas pequenas $(n=4)$} & $12,2 \pm 5,3$ \\
\hline & $\mathrm{KW}=1,64 \mathrm{~ns}$ \\
\hline
\end{tabular}




\begin{tabular}{lc}
\hline Artrópodes & \\
\hline \hline Densidade média de artrópodes por AF & $\mathbf{1 , 3}$ \\
$\mathrm{n}^{\circ}$ de abrigos com artrópodes & $\mathbf{2 3 ( 7 6 , 6 \% )}$ \\
$\mathrm{n}^{0}$ de abrigos sem artrópodes & $\mathbf{7 ( 2 3 , 4 \% )}$ \\
Abrigos apenas com um indivíduo & $\mathbf{2}$ \\
Abrigo apenas com Gonioterma sp. & $\mathbf{2}$ \\
\hline
\end{tabular}

Valores absolutos () e média \pm desvio-padrão. Diferentes letras entre as linhas indicam diferenças estatísticas $(P<0,05)$. Árvores grandes ( $>2 \mathrm{~m})$, árvores médias (1 a $2 \mathrm{~m})$ e árvores pequenas $(<1 \mathrm{~m})$.

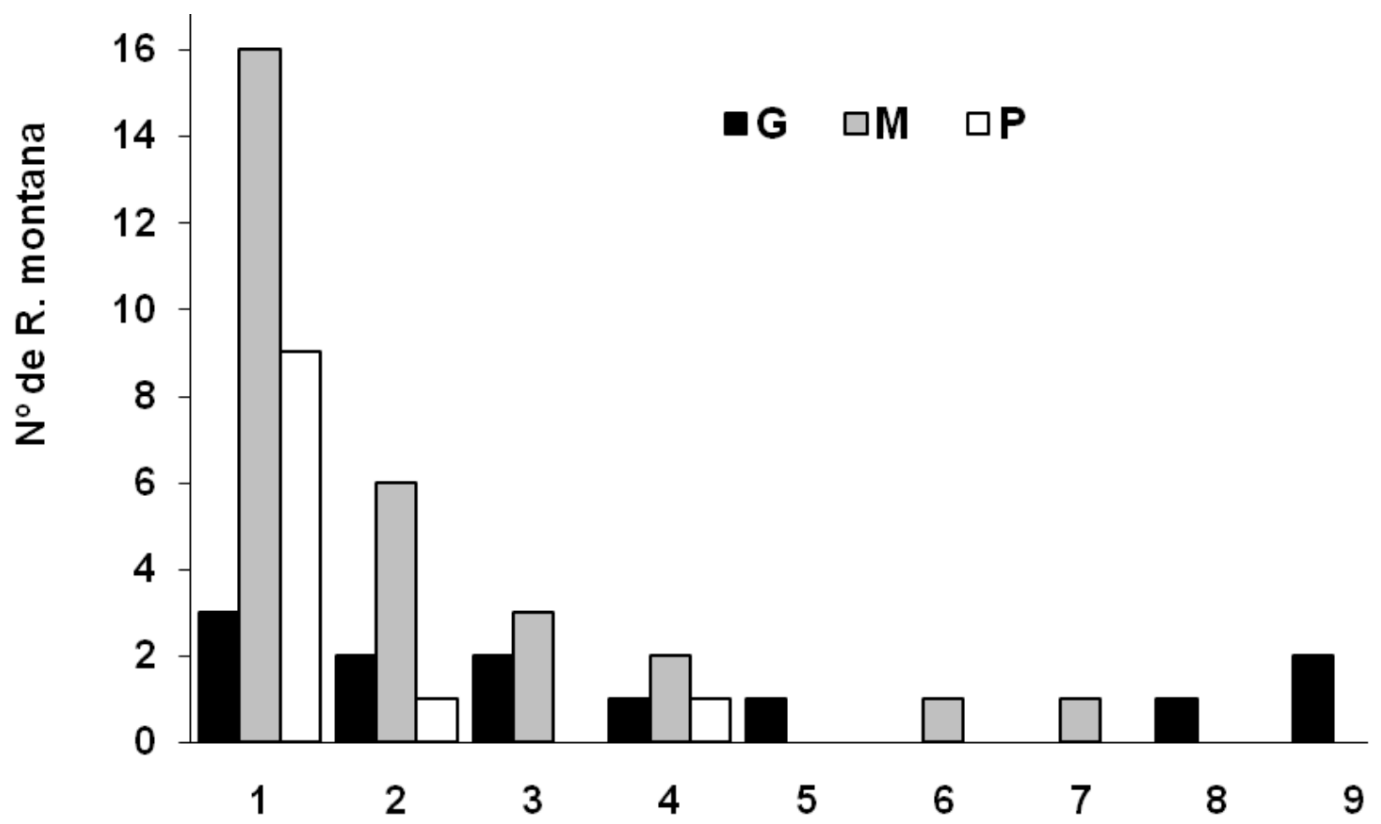

Figura 1 - Relação do número de abrigos foliares de Gonioterma sp. (Lepidoptera) nas diferentes classes de tamanho de R. montana (Proteaceae), em vegetação de cerrado, no Parque Estadual de Caldas Novas, GO. As classes de tamanho foram: $\mathrm{P}=$ pequena (plantas com altura menor que $1 \mathrm{~m}$ ); $\mathrm{M}=$ média (plantas com alturas maiores que $1 \mathrm{~m}$ e menores que $2 \mathrm{~m}$ ) e $\mathrm{G}=$ grande (plantas com altura superior à $2 \mathrm{~m}$ )

\section{COMPOSIÇÃO, ABUNDÂNCIA E RIQUEZA DE ARTRÓPO- DES ASSOCIADOS AOS ABRIGOS FOLIARES}

As lagartas de Gonioterma sp. utilizam em média 10 folhas para construir o seu abrigo, independentemente do tamanho da planta hospedeira (Tabela 1). A maioria dos AFs (76\%) apresentou artrópodes em seu interior e em apenas $40 \%$ dos abrigos foram encontradas larvas de Gonioterma sp. Em dois AFs foi registrado um único artrópode residente e em outros dois AFs, apenas a larva de Gonioterma sp. (Tabela 1).

Foram registradas 43 morfoespécies e uma abundância de 81 indivíduos de artrópodes nos 30 AFs amostrados. Do total, 33 morfoespécies $(81 \%)$ foram representadas por apenas um indivíduo (Tabela 2). A maior abundância ocorreu para uma morfoespécie da ordem Collembola (com 15 indivíduos), seguido pelo lepidóptero
Gonioterma sp. (com 12 indivíduos) e uma morfoespécie da ordem Blattodea (com seis indivíduos) (Tabela 2).

A riqueza de taxa foi representada por sete ordens e 11 famílias. Os táxons mais ricos em morfoespécies foram Hymenoptera, com 11 morfoespécies (sendo 10 morfoespécies de Formicidae), Arachnida, com 11 morfoespécies (sendo Salticidae a família mais representativa) e Coleoptera, com oito morfoespécies (Tabela 2 ). As guildas mais ricas em espécies foram de predadores (23 morfoespécies) seguidos de herbívoros sugadores (12 morfoespécies), herbívoros mastigadores e insetos saprófagos (cada um com três morfoespécies). Já os grupos com maior número de indivíduos foram os predadores (27 ind.), seguidos pelos saprófagos (26 ind.), herbívoros mastigadores (17 ind.) e herbívoros sugadores (12 ind.) (Tabela 2). 
Tabela 2 - Artrópodes registrados no interior de 30 abrigos foliares construídos por Gonioterma sp. (Lepidoptera) em folhas de R. montana (Proteaceae) de vegetação de cerrado no Parque Estadual de Caldas Nova, GO.

\begin{tabular}{|c|c|c|c|}
\hline Táxons & Riqueza & Abundância & Guilda \\
\hline \multicolumn{4}{|l|}{ Arachnida } \\
\hline Oxyopidae & 1 & 2 & Predador \\
\hline Araneidae & 4 & 4 & Predador \\
\hline Lycosidae & 1 & 1 & Predador \\
\hline Salticidae & 5 & 7 & Predador \\
\hline \multicolumn{4}{|l|}{ Coleoptera } \\
\hline Coleoptera & 5 & 5 & Herb.* Mastigador \\
\hline Meloidae & 1 & 1 & Herb. Mastigador \\
\hline Bruchidae & 2 & 2 & Herb. Mastigador \\
\hline Collembola & 1 & 15 & Saprófago \\
\hline Diptera & 1 & 4 & Saprófago \\
\hline \multicolumn{4}{|l|}{ Hemiptera } \\
\hline Homoptera & 2 & 2 & Herb. Sugador \\
\hline Dactylopiidae & 1 & 1 & Herb. Sugador \\
\hline Aphidoidea & 1 & & Herb. Sugador \\
\hline \multicolumn{4}{|l|}{ Hymenoptera } \\
\hline Hymenoptera sp. & 1 & 1 & Predador \\
\hline Formicidae & 11 & 12 & Predador \\
\hline \multicolumn{4}{|l|}{ Lepidoptera } \\
\hline \multicolumn{4}{|l|}{ Elachistidae } \\
\hline Goniotherma & 1 & 12 & Herb. Mastigador \\
\hline Lepidoptera & 3 & 5 & Herb. Mastigador \\
\hline \multicolumn{4}{|l|}{ Orthoptera } \\
\hline Blattodea & 2 & 7 & Saprófago \\
\hline TOTAL & 43 & 81 & 4 \\
\hline
\end{tabular}

*Herb. hábito alimentar herbívoro.

RELAÇÕES ENTRE A ALTURA DA PLANTA E AS GUILDAS DE ARTRÓPODES NOS ABRIGOS FOLIARES

A análise entre a associação das categorias de altura da planta hospedeira com a abundância das guildas e de larvas de Gonioterma sp. demonstrou autovalores com elevadas porcentagens. $\mathrm{Na}$ de composição da inércia total, o eixo 1 explicou 94,5\% e o eixo 2 explicou $0,5 \%$ da variabilidade total (Tabela 3). A elevada significância do teste qui-quadrado no modelo rejeitou a hipótese nula de independência de associação entre as variáveis (linha e colunas), que significa desvios significativos dos perfis linha e coluna em relação ao seu centroide. O teste revelou, portanto, que as variáveis apresentaram um considerável grau de associação (Tabela 3).

Tabela 3 - Valores dos parâmetros fornecidos pelo modelo de análise de correspondência para associação entre o tamanho das plantas de R. montana com a densidade de artrópodes no interior de AFs de Gonioterma $s p$., em vegetação de cerrado no Parque Estadual de Caldas Novas, GO.

\begin{tabular}{ccccc}
\hline Dimensões & Inércia & Autovalor & Qui-quadrado & Proporção explicada \\
\hline 1 & 0,052 & 0,228 & & $94,5 \%$ \\
2 & 0,003 & 0,055 & & $0,5 \%$ \\
\hline Total & 0,055 & & $23,82 * * *$ & $100 \%$ \\
\hline
\end{tabular}


A representação da associação no mapa perceptual revelou que as plantas de porte pequeno (até $1 \mathrm{~m}$ de altura) estiveram mais associadas com a guilda de artrópodes saprófagos, enquanto as plantas de porte médio com as guildas de predadores e herbívoros mastigadores, e as plantas de maior porte (altura $>2 \mathrm{~m}$ ) com herbívoros sugadores. Os AFs com larvas e pupas de Gonioterma sp. foram registrados em plantas de porte médio (Figura 2).

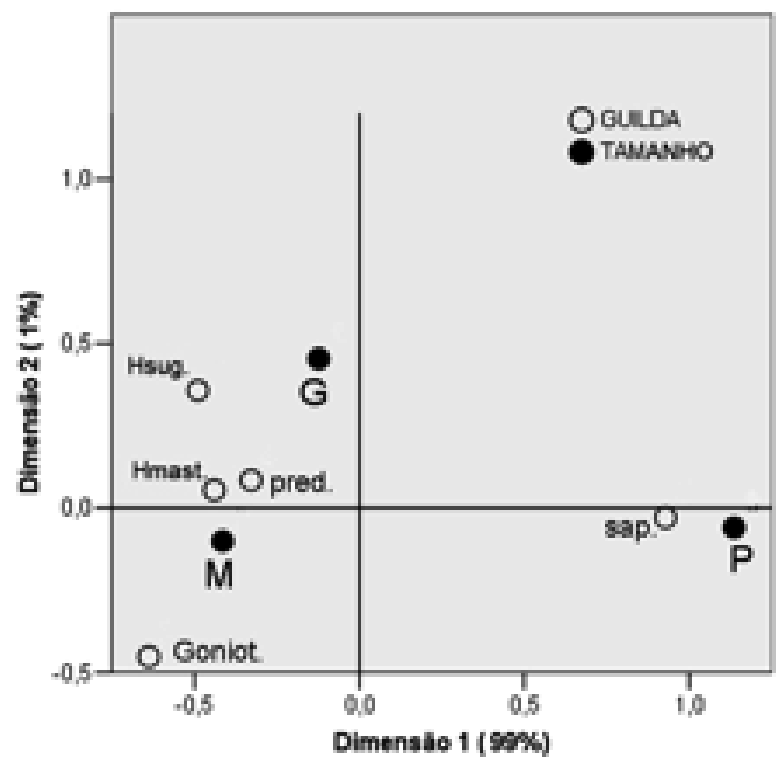

Figura 2 - Mapa perceptual da análise de correspondência demonstrando as relações entre o tamanho das plantas de $R$. montana com a abundância de artrópodes no interior de AFs de Gonioterma sp., em vegetação de cerrado no Parque Estadual de Caldas Novas, GO. As categorias de altura das plantas foram: grande $(G)(h>2 m)$, médio ( $M)(1 \mathrm{~m} \leq \mathrm{h}<2 \mathrm{~m})$ e pequeno $(P)(0,4 \mathrm{~m}<\mathrm{h}<1 \mathrm{~m})$. Os artrópodes foram categorizados como larvas de Gonioterma sp. (Goniot.), lepidóptero construtor do abrigo, e guildas de herbívoros mastigadores (Hmast.), predadores (pred.), saprófagos (sap.) e herbívoros sugadores (Hsug.).

\section{Discussão}

RELAÇÃO ENTRE ALTURA DE $R$. MONTANA E ABUNDÂNCIA DE AFS

Os resultados demonstraram elevada frequência e abundância relativas de AFs nas plantas $R$. montana de maior porte. De acordo com Marquis et al. (2002), plantas mais altas podem ser colonizadas por um maior número de mariposas pelo simples fato de apresentarem maior quantidade de recursos. De modo geral, a variação entre os indivíduos de uma planta hospedeira afeta a disponibilidade e a qualidade de recursos para os herbívoros, sendo, a idade da planta, o genótipo e a influência do ambiente os três principais geradores desta variabilidade
(Hunter \& Price, 1998). Alternativamente, plantas de maior porte podem ser mais facilmente localizadas pelo lepidóptero, aumentando as chances de que as plantas mais altas sejam selecionadas para a oviposição (e.g. Wiklund \& Frigerg, 2009). Por isso, as plantas pequenas, com a copa ainda não formada, podem não fornecer condições adequadas para a mariposa depositar seus ovos, ou não serem prioritárias durante o processo de escolha.

\section{COMPOSIÇÃO, ABUNDÂNCIA E RIQUEZA DE ARTRÓ- PODES ASSOCIADOS AOS AFS}

No interior dos AFs de Gonioterma sp. foi observada significativa diversidade de artrópodes, porém com baixa abundância por espécie. Segundo Martinsen et al. (2000), os abrigos foliares oferecem proteção, sendo esta característica o principal atrativo para outros artrópodes. Além da proteção contra inimigos naturais, os AFs podem conferir também proteção contra fatores abióticos adversos, como insolação e chuvas fortes. Além disso, os AFs podem manter temperaturas mais amenas e umidade mais elevada (Lill \& Marquis, 2007). Tais condições favorecem a ocupação dos AFs por uma grande variedade de guildas, tais como os insetos herbívoros sugadores (Fukui, 2001; Nakamura e Ohgushi, 2003). Embora a colonização dos AFs possa estimular as relações predador-presa entre os artrópodes residentes (Larsson et al., 1997; Lill et al., 2007), não foi possível indicar se estas interações ocorreram no presente estudo.

Foi observado considerável variação no número de guildas de artrópodes utilizando os AFs de Gonioterma sp., principalmente por grupos de artrópodes predadores, herbívoros ou detritívoros. Além disso, é possível que os abrigos foliares sejam também utilizados como alimento por essas guildas de insetos herbívoros e detritívoros.

A construção dos AFs e as modificações das condições microclimáticas pode determinar a modificação da estrutura da comunidade de artrópodes, atribuindo às lagartas produtoras dos abrigos o papel de engenheiros de ecossistema (Lill \& Marquis, 2007). Os abrigos produzidos pela mariposa Anacampsis niveopulvella (Gelechiidae), em híbridos de algodão (Populus angustifolia X P. fremontii), também estimulam aumento na riqueza e abundância de outros artrópodes, principalmente de herbívoros e predadores (Martinsen et al., 2000).

As aranhas são predadores comumente encontrados em AFs (Damman, 1993; Fukui, 2001). No presente estudo foi amostrada considerável abundância de formigas e aranhas no interior dos AFs de Gonioterma sp. em $R$. montana. 
Por fim, a diversidade amostrada também pode estar relacionada às características da planta hospedeira, que apresenta uma ampla distribuição no bioma Cerrado e, como observado, pode ocorrer em diferentes comunidades vegetais. Espécies vegetais com estas características tendem abrigar uma maior diversidade de fauna de invertebrados associadas do que plantas que apresentam uma distribuição geográfica limitada ou restrita a poucos ambientes (Hayson \& Coulson, 1998).

As RELAÇÕES ENTRE A ALTURA DA PLANTA E AS GUILDAS DE ARTRÓPODES NOS ABRIGOS FOLIARES

Os mecanismos que condicionam a preferência dos animais por uma determinada espécie de planta ou partes das mesmas podem ser condicionados também por pressões seletivas de competição por recursos e predação (Rabenold \& Bromer, 1988). A análise de correspondência indicou a tendência de especialização da localização do AF nas plantas (sua altura) por parte dos artrópodes. Os artrópodes saprófagos, por exemplo, estariam mais relacionados com as plantas pequenas ( $<1 \mathrm{~m}$ de altura). Esse grupo foi representado pelos Collembola, um grupo de artrópodes numericamente dominante no solo e serapilheira e que servem de alimento para outros invertebrados (Hopkin, 1997). As plantas de maior porte ( $>2 \mathrm{~m}$ de altura) estiveram relacionadas com a presença de herbívoros sugadores, secundariamente, por herbívoros mastigadores e predadores. Os predadores, por sua vez, foram mais relacionados com plantas de porte médio.

Geralmente, os artrópodes herbívoros preferem locais onde há maior disponibilidade de recursos, ou podem se concentrar em determinadas áreas devido a previsibilidade do recurso alimentar, ou pela facilidade de localização da planta hospedeira (Franklin, 1970; Wiklund \& Frigerg, 2009). Da mesma forma, isso ocorreu com a presença de predadores, onde sua abundância estaria relacionada diretamente com o potencial de presas existentes (Martinsen et al., 2000).

O presente estudo demonstrou a relação entre a altura de $R$. montana com algumas características dos abrigos foliares de Gonioterma sp. presentes nas copas dessa planta hospedeira. O papel exercido por Gonioterma sp. como engenheiro de ecossistema ficou destacado pela importância dos abrigos foliares produzidos por este animal como refúgios para a fauna de artrópodes local. A densidade de AFs e a composição de espécies de artrópodes no interior desses foram relacionadas com a altura da planta. Este resultado reflete a complexidade das relações entre engenheiros de ecossistemas com o ambiente e a diversidade local, enfatizando a heterogeneidade ambiental e seu papel para manutenção da diversidade na comunidade.

\section{Agradecimentos}

Os autores agradecem à administração do PESCAN por ceder a estrutura para a pesquisa. Aos profs. Dra Helena C. de Moraes (Universidade de Brasília-UnB), Dr Paulo Eugênio Oliveira e Dra Kátia G. F. Giaretta (Universidade Federal de Uberlândia-UFU) pelo auxilio na execução do estudo e revisão crítica da primeira versão deste trabalho. Ao Dr. Kleber Del-Claro (UFU) pelo uso dos equipamentos em seu laboratório. À CAPES pela bolsa de mestrado ao primeiro autor (2005-2007). Aos revisores anônimos pela contribuição na redação do manuscrito. Esse estudo fez parte do relatório do curso de campo do PPG Ecologia e Conservação de Recursos Naturais da UFU.

\section{ReferênCIAS}

Bendicho-López A.; H.C. Morais; J.D. Hay \& I.R. Diniz. 2006. Lepidópteros folívoros em Roupala montana Aubl. (Proteaceae) no Cerrado sensu stricto. Neotropical Entomology 35: 182-191.

Costa, A.A. \& E.M. Varanda. 2002. Building of leaf shelters by Stenoma scitiorella Walker (Lepidoptera: Elachistidae): manipulation of host plant quality? Neotropical Entomology 31: 537-540.

Damman, H. 1993. Patterns of interaction among herbivores species. p. 132-169. In: N.E. STAMP, T.M. CASEY (Eds.). Caterpillars: ecological and evolutionary constraints on foraging. Chapman and Hall, New York.

Diniz, I.R. \& H.C. Morais. 1995. Larvas de Lepidoptera e suas plantas hospedeiras em um cerrado de Brasília, DF, Brasil. Revista Brasileira de Entomologia 39:755-770.

Diniz, I.R. \& H.C. Morais. 1997. Lepidopteran caterpillar fauna on cerrado host plants. Biodiversity Conservation 6:817-836.

Diniz, I.R. \& H.C. Morais. 2002. Local pattern of host plant utilization by lepidopteran larvae in the cerrado vegetation. Entomotropica 17:115-119.

Diniz, I.R.; H.C. Morais; A. Botelho; F. Venturoli \& B. C. Cabral. 1999. Lepidopteran caterpillar fauna on lactiferous host plants in the central Brazilian cerrado. Revista Brasileira de Biologia 59:627-635.

Embrapa. 1982. Levantamento de reconhecimento de média intensidade dos solos e avaliação da aptidão agrícola das terras do 
Triângulo Mineiro. Ministério da Agricultura e do Abastecimento, Rio de Janeiro.

Felfili, J.M. 1997. Dynamics of the natural regeneration in the Gama gallery forest in central Brazil. Forest Ecology and Management 9:235-245.

Fornier, V.; J.A. Rosenhein; J. Brodeur; L.O. Laney \& M.W. Johnson. 2003. Herbivorous mites as ecological engineers: indirect effect on arthropods inhabiting papaya foliage. Oecologia 135:442-450.

Franklin, R.T. 1970. Insect influences on the forest canopy. p. 86-99. In D.E. REICHLE (Ed.). Analysis of temperate forest ecosystems. Springer, Berlin.

Fukui, A. 2001. Indirect interaction mediated by leaf shelters in animal-plant communities. Population Ecology 43:31-40.

Hayson, K.A. \& J.C. Coulson. 1998. The Lepidoptera fauna associated with Calluna vulgaris: effect of plant architecture on abundance and diversity. Ecological Entomology 23:377-385.

Heppner, J.B. 1984. Atlas of Neotropical Lepidoptera checklist, MicropterigoideaImmoidea. Part. I. Junk Publishers, Boston. $112 p$.

Hopkin, S.P. 1997. Biology of the Springtails (Insecta: Collembola). Oxford University Press, New York.

Hunter, M.D. \& P.W. Price. 1998. Natural variability in plants and animals. p. 1-12. In M.D. HUNTER; T. OHGUSHI; P.W. PRICE (Ed.). Effects of resources distribution on animal-plant interactions. Academic Press, New York.

Jones, T.G.; I. Castellanos \& M.R. Weiss. 2002. Do leaf shelter always protect caterpillar from invertebrate predators? Ecological Entomology 27:753-757.

Köppen, W. 1948. Climatologia: con un estudio de los climas de la tierra. Fondo de Cultura Econômica. México. 479p.

Larsson, S.; H. E. Haggstrom \& R. F. Denno. 1997. Preference for protected feeding sites by larvae of the willow-feeding leaf beetle Galerucella lineola. Ecological Entomology 22:445-452.

Lill, J.T. \& R.J. Marquis. 2007. Microhabitat manipulation: ecosystem engineering by shelter-building insects. In Ecosystem Engineers: Plants to Protists (CUDDINGTON,
K.; BYERS, J.E.; WILSON, W.G.; HASTINGS, A. Eds.). pp: 107-138. Elsevier Academic Press

Lill, J.T.; R.J. Marquis; M. Walker \& L. Peterson. 2007. Ecological consequences of shelter-sharing by leaf-tying caterpillars. Entomological Experimentalis et Applicata: 1-9.

Marquis, R.J.; J.T. Lill \& A. Piccini. 2002. Effect of plant architecture on colonization and damage by leaftying caterpillars of Quercus alba. Oikos 99:531-537.

Martinsen, G.D.; K.D. Float; A.M. Waltz; G.M. Wimp \& T.G. Whithan. 2000. Positive and negative interaction between leaf rollers and the others arthropods enhance biodiversity hybrid cottonwoods. Oecologia 136:445449.

Morais, H.C.; I.M. Mahajan \& I.R. Diniz. 2005. História natural da mariposa Chlamydastis smodicopa (Meyrick) (Lepidoptera, Elachistidae, Stenomatinae). Revista Brasileira de Zoologia 22:633-638.

Nakamura, M. \& T. Ohgushi. 2003. Positive and negative effects of leaf shelters on herbivorous insects: Linking multiple herbivore species on a willow. Oecologia 136:445-449.

Rabenold, K.N. \& W.R. Bromer. 1988. Plant communities as animal habitats: effects of primary resources on the distribution and abundance of animals. p. 291-353. In W. ABRAHAMSON (Ed.). The ecology of plantanimal interactions. McGraw-Hill, New York.

Wiklund, C. \& M. Frigerg. 2009. The evolutionary ecology of generalization: among-year variation in host use and offspring survival in a butterfly. Ecology 90:3.406-3.417

Recebido em 26.IX.2013 Aceito em 09.IV.2014 